\title{
Evaluación de la utilidad de la prueba rápida de casete por inmunocromatografía para el diagnóstico de dengue en una región endémica colombiana
}

\author{
Ruth Aralí Martínez-Vega, Fredi Alexander Díaz-Quijano, Carolina Coronel-Ruiz, Sergio Yebrail \\ Gómez, Luis Ángel Villar-Centeno
}

Grupo de Epidemiología Clínica, Centro de Investigaciones Epidemiológicas, Facultad de Salud, Universidad Industrial de Santander, Bucaramanga, Colombia

Introducción. Las pruebas convencionales para el diagnóstico serológico de dengue son de reducida utilidad para el clínico durante los primeros días de la enfermedad, debido a su baja sensibilidad, su relativa complejidad técnica y el tiempo necesario para su realización.

Objetivo. Determinar la reproducibilidad, exactitud y utilidad clínica de la prueba rápida en casete (PANBIO) para el diagnóstico de dengue en muestras de pacientes agudos o convalescientes.

Materiales y métodos. La prueba de inmunocromatografía se evaluó en pacientes de 5 o más años de edad, con síndrome febril agudo, captados en el área metropolitana de Bucaramanga entre abril y agosto de 2003. Se utilizó suero de la fase aguda y de la convalecencia de 67 casos de dengue diagnosticados mediante pruebas de IgM pareadas (MAC-ELISA), y de 33 con síndrome febril agudo de etiología diferente a dengue.

Resultados. La prueba mostró buena reproducibilidad entre observadores (kappa=0,84). En la convalecencia, la prueba para la IgM tuvo una sensibilidad de $76,1 \%$, especificidad de $75,8 \%$, un valor diagnóstico positivo de $86,4 \%$, un valor diagnóstico negativo de $61 \%$, una razón de verosimilitud positiva de 3,14 y una negativa de 0,32 . En la fase aguda, los valores encontrados fueron $52,2 \%, 84,8 \%, 87,5 \%, 46,7 \%, 3,45$ y 0,56 , respectivamente.

Conclusiones. La prueba de casete para el diagnóstico rápido de dengue muestra una buena reproducibilidad y unas características operativas aceptables. Por esta razón, podría recomendarse como ayuda diagnóstica en áreas endémicas de dengue.

Palabras clave: dengue/diagnóstico, técnicas y procedimientos diagnósticos, sensibilidad y especificidad, reproducibilidad de resultados.

Evaluation of PANBIO rapid immunochromatographic cassette for dengue diagnosis in a Colombian endemic area

Introduction. Traditional tests for dengue diagnosis are not useful during the first days of disease onset, because they require time and complex techniques or because they have low sensitivity. Objective. The reliability and precisison was determined for the rapid test in cassette (PANBIO) for dengue diagnosis in acute and convalescent serum samples.

Materials and methods. The immunochromatographic test was evaluated in samples from patients older than 5 years with acute febrile syndrome. The patients came from the metropolitan area of Bucaramanga during April and August of 2003. Acute and convalescent serum came from 67 dengue cases, diagnosed by viral isolation or IgM paired tests (modified MAC-ELISA), and from 33 patients with acute febrile syndrome (not dengue) that served as controls.

Results. Reliability was good (kappa=0.84). In the convalescent serum, the test showed sensitivity of $76.1 \%$, specificity of $75.8 \%$, positive predictive value of $86.4 \%$ and negative predictive value of $61.0 \%$, positive likelihood ratio 3.14 and negative likelihood ratio 0.32 . In the acute phase, the values were $52.2 \%, 84.8 \%, 87.5 \%, 46.7 \%, 3.45$ and 0.56 , respectively.

Conclusions. Rapid immunochromatographic test for dengue diagnosis shows good reproducibility and specificity, with an acceptable sensitivity. Therefore, this rapid test is recommended as diagnostic tool in endemic areas. 
Key words: Dengue/diagnosis, diagnostic techniques and procedures, sensitivity and specificity, reproducibility of results.

El dengue es la enfermedad viral transmitida por vectores más frecuente en el mundo (1). En América, durante el 2008, se reportaron a la Organización Mundial de la Salud (OMS) 908.926 casos probables de dengue (58.521 confirmados), 25.696 casos de dengue hemorrágico y 306 muertes. Durante este mismo año, Colombia ocupó el cuarto lugar del continente, con el reporte de 3.081 casos de dengue hemorrágico/síndrome de choque por dengue y 12 muertes (2). El área metropolitana de Bucaramanga está conformada por cuatro de los trece municipios hiperendémicos del país (3).

La identificación temprana de la fiebre del dengue es un pilar para la prevención y la atención oportuna de sus complicaciones, que ocurren usualmente hacia el quinto día de enfermedad $(4,5)$. Por ello, la demora en su diagnóstico puede tener implicaciones sobre el pronóstico, puesto que el inapropiado manejo de estos pacientes en los primeros días puede ser un factor determinante de la aparición de complicaciones potencialmente letales $(6,7)$.

Sin embargo, el diagnóstico clínico de dengue en los primeros días de enfermedad es difícil (8), dado que se presenta como un síndrome febril agudo con síntomas inespecíficos; además, su diagnóstico diferencial comprende una amplia variedad de entidades, entre las cuales están enfermedades como la influenza, la rubéola, la gastroenteritis, la fiebre tifoidea, la fiebre amarilla, el paludismo y la leptospirosis (9-11). Lo anterior genera una alta discordancia entre el diagnóstico clínico y el de laboratorio, que indica la importancia de confirmar la infección por dengue con pruebas de laboratorio $(8,12,13)$.

Los métodos empleados en la confirmación del dengue incluyen el aislamiento viral, la

\footnotetext{
Correspondencia:

Ruth Aralí Martínez-Vega, Centro de Investigaciones Epidemiológicas, Facultad de Salud, Universidad Industrial de Santander, Carrera 32 № 29-31, tercer piso, oficina 304, Bucaramanga, Colombia.

Telefax: (7) 6345781

Recibido: 16/03/09; aceptado:17/06/09
}

detección de anticuerpos por la técnica ELISA o la inhibición de la hemaglutinación, pruebas que, generalmente, son informadas por el laboratorio a los servicios de salud entre 8 y 15 días después de la toma de la muestra. Asimismo, otras técnicas, como la reacción en cadena de la polimerasa (PCR), la inmunohistoquímica en casos fatales o la inmunofluorescencia, son de alta complejidad para el medio y de costo elevado $(12,14)$.

Además, las anteriores pruebas requieren un laboratorio especializado y personal entrenado, lo que limita su disponibilidad en la mayoría de las áreas endémicas. Por estas razones, en la práctica clínica la utilidad de estos procedimientos es limitada para obtener resultados oportunos, para la toma de decisiones en el manejo del paciente en las horas siguientes a su consulta. Considerando lo anterior, la OMS ha resaltado la importancia de desarrollar procedimientos diagnósticos rápidos, aplicables en la primera evaluación del síndrome febril agudo en áreas endémicas de dengue.

En los últimos años se ha evaluado una prueba presuntiva de diagnóstico rápido por inmunocromatografía en tira (PANBIO®, Rapid Dengue Test), cuya realización es sencilla, ágil (tarda menos de una hora) y no requiere equipos ni laboratorio especializados (1521). Sin embargo, a pesar de sus ventajas, se ha registrado una gran variación en las características operativas de la prueba. Su sensibilidad ha oscilado entre $45 \%$ y $100 \%$, y su especificidad entre $57 \%$ y $100 \%$, variaciones que se han atribuido principalmente al momento de la enfermedad en el que se toman las muestras (22).

Una presentación nueva de esta prueba es el casete, que emplea una menor cantidad de suero o sangre total y no requiere dilución de la muestra. Esta prueba, de menor complejidad, podría aplicarse durante la consulta inicial y orientar las intervenciones tempranas del síndrome febril agudo. Sin embargo, sus características operativas no se han evaluado. Por tal razón, 
el objetivo del presente estudio fue determinar la reproducibilidad entre observadores, la exactitud (sensibilidad, especificidad y valores diagnósticos) y la utilidad clínica (razón de verosimilitud, likelihood ratio) de la prueba rápida de inmunocromatografía en casete para el diagnóstico temprano del dengue.

\section{Materiales y métodos}

Para la evaluación de esta prueba diagnóstica se hizo un muestreo transversal. Se emplearon sueros almacenados de todos los pacientes mayores de cinco años con síndrome febril agudo de causa inaparente, que consultaron a dos centros de atención en salud (Centro de Salud Rosario y en la Fundación Oftalmológica Clínica Carlos Ardila Lulle-FOSCAL) del área metropolitana de Bucaramanga (Colombia), entre abril y agosto de 2003.

La captación se realizó por medio de una llamada telefónica a los pacientes reportados diariamente por los centros. En el caso del Centro de Salud Rosario, los sujetos con síndrome febril agudo eran atendidos en el servicio de urgencias; en la FOSCAL, los pacientes consultaban tanto a urgencias como al servicio de consulta oportuna, o prioritaria, de la institución.

Una vez que los pacientes autorizaron telefónicamente, fueron visitados en su domicilio, donde aceptaron su participación en el estudio mediante la firma (o la de su acudiente) de un consentimiento informado, previamente revisado y aprobado por el Comité de Ética de la Universidad Industrial de Santander.

Los criterios de inclusión fueron: individuos con edad mayor de 5 años, con fiebre de instalación abrupta abordados por el clínico en las primeras 96 horas de enfermedad (a partir del inicio de la fiebre).

Se excluyeron los pacientes que al momento del ingreso presentaban cualquiera de las siguientes condiciones: evidencia clínica de otro proceso infeccioso que explicara de forma parcial o total la enfermedad actual (amigdalitis, celulitis, neumonía, etc.), diabetes, sida, cirrosis, enfermedades reumatológicas o tumorales, insuficiencia cardiaca o renal, o historia de ingestión de corticoides, residencia en zona rural o de difícil acceso para el seguimiento $y$, finalmente, aquéllos en los que no había disponibilidad de suero de la fase aguda y de fase convaleciente, refrigerado para la realización de la prueba en casete.

\section{Muestras}

De cada individuo se obtuvo una muestra de sangre en las primeras 96 horas del síndrome febril agudo (suero de fase aguda), momento en el cual se registraron las características clínicas de los pacientes. Además, después del séptimo día de enfermedad, se obtuvo una segunda muestra de suero (suero de fase convaleciente). Todas las muestras se almacenaron en un refrigerador a $-70^{\circ} \mathrm{C}$ hasta la realización de las pruebas diagnósticas.

\section{Método de referencia}

Para documentar o descartar la infección por el virus del dengue, se evaluaron los niveles de lgM en todas las muestras de fase convaleciente, utilizando un sistema de ensayo de captura (MAC-ELISA) adaptado en el Laboratorio de Virología del Centro de Investigaciones en Enfermedades Tropicales de la Universidad Industrial de Santander (CINTROP-UIS) $(23,24)$. La prueba se consideró positiva cuando el valor de absorbancia fue, al menos, el doble de la media de los controles negativos más dos desviaciones estándar.

Para estimar la variación en los títulos de anticuerpos, se realizó la prueba en suero de fase aguda de aquellos pacientes que tuvieron IgM positiva en el suero de fase de convalecencia.

La infección aguda por dengue se consideró confirmada, según la recomendación de la OMS (12), cuando se evidenció seroconversión (IgM de suero de fase aguda negativa e IgM de suero de fase convaleciente positiva) o cuadruplicación de los títulos de anticuerpos. Se consideró un síndrome febril agudo de otra etiología (no dengue), cuando la prueba de lgM específica contra dengue en la muestra de fase convaleciente fue negativa. 


\section{Prueba de inmunocromatografía}

La prueba de diagnóstico rápido de dengue en casete (Dengue Duo IgM and IgG Rapid Cassette, PANBIOß) se realizó en los sueros de fase aguda y de fase convalecencia de los pacientes incluidos en el estudio.

De acuerdo con las recomendaciones de la casa matriz, se utilizaron $10 \mu \mathrm{l}$ de suero y se leyó la prueba a los 15 minutos de montaje. Se consideró como positiva, para anticuerpos IgM e lgG y para el control, la aparición de una línea rosada de cualquier intensidad, al frente de cada letra de referencia que trae el casete $(M, G$ y $C$, respectivamente) (figura 1).

En todos los casos, la lectura fue hecha por dos observadores independientes, quienes desconocían el resultado de la prueba de referencia. En consideración al interés de establecer la reproducibilidad de la lectura de la prueba realizada, por cualquier miembro del equipo de salud, independientemente de su nivel de entrenamiento, uno de los observadores fue un bacteriólogo y el otro un médico.

Los casos considerados dudosos se leyeron nuevamente a los 30 minutos de montada la prueba (de acuerdo con las instrucciones de la casa matriz). Cuando no existió concordancia entre las lecturas de los observadores, se recurrió a un tercer evaluador, quien también desconocía el resultado de la prueba ELISA, y se decidió el resultado teniendo en cuenta la concordancia entre dos de las tres lecturas. Se repitió la prueba cuando no apareció la línea control, cuando persistió la duda después de la segunda lectura a los 30 minutos y cuando no hubo concordancia entre dos de los tres evaluadores.

\section{Almacenamiento y análisis de datos}

La información se almacenó en una base de datos electrónica (Microsoft Excel 97, Microsoft Corp). Se describieron los datos demográficos de la población en los pacientes con dengue y síndromefebrilagudo nodengue.Posteriormente, se determinaron la reproducibilidad entre observadores (kappa), la sensibilidad, la especificidad y los valores diagnósticos positivo

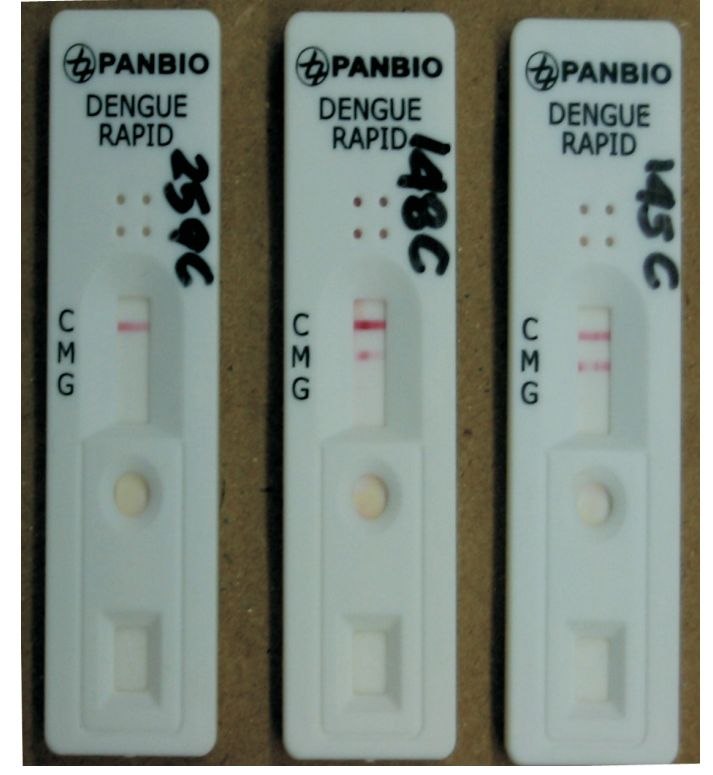

Figura 1. Prueba rápida de casete por inmunocromatografía (PANBIO®). Se observan: a la izquierda, prueba negativa para IgM e IgG; en el centro y a la derecha, pruebas positivas para lgM.

y negativo y las razones de verosimilitud (likelihood ratio) positivas y negativas de la prueba de casete para el diagnóstico de dengue, tanto en suero de fase aguda como en el tomado en la fase de convalecencia.

Además, en lospacientescondengueconfirmado, se evaluó la asociación entre el resultado de la IgM por inmunocromatografía y las variables del paciente, como tiempo de enfermedad al momento de la recolección de la muestra, fiebre al examen físico, el resultado de la prueba de aislamiento viral y la identificación de $\lg G$ en la misma prueba de PANBIO. El análisis se realizó empleando el programa estadístico Stata SE versión 9.2.

\section{Resultados}

Durante el periodo de mayo a agosto de 2003, se captaron 111 pacientes con historia de síndrome febril agudo, de los cuales, 100 se pudieron incluir en el estudio porque tenían muestras pareadas refrigeradas. Los 11 pacientes no incluidos fueron 4 casos de dengue confirmado y 7 de fiebre no dengue (IgM negativos). De los 100 pacientes incluidos, a 67 pacientes se les 
confirmó la infección por el virus dengue y 33 pacientes se consideraron como síndrome febril agudo de otra etiología.

Cuando se compararon los grupos de pacientes con dengue y síndrome febril agudo no dengue, no se evidenciaron diferencias estadísticamente significativas para las variables de edad, sexo, ni presencia de fiebre al examen físico de ingreso. Aunque no fue significativa la diferencia del promedio de las horas de la enfermedad entre los grupos $(p=0,05)$, los pacientes con dengue presentaron en promedio más tiempo de evolución al ingreso (cuadro 1).

Se realizaron 200 pruebas $\mathrm{PANBIO} \AA_{\text {rápidas }}$ de casete (100 en muestra de fase aguda y 100 en muestra de fase convaleciente). Para su interpretación, 4 (2,0\%) requirieron una segunda lectura, se definió el resultado con un tercer evaluador en $9(4,5 \%)$ y se repitieron $16(8,0 \%)$, cuatro de éstas últimas porque no presentaron la línea control (figura 2).

La reproducibilidad entre observadores de la prueba de casete para la lgM y para la IgG fue alta (kappa de 0,84, y de 0,78), encontrándose un porcentaje de acuerdo observado de $91,3 \%$ y de $88,8 \%$, respectivamente. Además, al evaluar la reproducibilidad en los grupos según el método diagnóstico de referencia, se observó un porcentaje de acuerdo de $92,4 \%$ en el grupo con diagnóstico confirmado de dengue y de $89,1 \%$ en el de no dengue.

Teniendo en cuenta el resultado de la lgM, la prueba de inmunocromatografía exhibió una sensibilidad de $29,4 \%$ antes de las primeras 72 horas, la cual aumentó a $60,0 \%$ cuando la muestra se tomó después de las 72 horas de enfermedad. Finalmente, la sensibilidad más alta fue de $76,1 \%$, documentada en la muestra

Cuadro 1. Características generales de los pacientes.

\begin{tabular}{|c|c|c|c|c|}
\hline Variable & $\begin{array}{c}\text { Total } \\
(n=100)\end{array}$ & $\begin{array}{c}\text { Dengue } \\
(n=67)\end{array}$ & $\begin{array}{c}\text { Síndrome febril agudo } \\
\text { no dengue } \\
(n=33)\end{array}$ & $\mathbf{P}$ \\
\hline \multicolumn{5}{|l|}{ Al ingreso al estudio } \\
\hline Horas de enfermedad Media $\pm \mathrm{DE}$ & $70,8 \pm 20$ & $73,6 \pm 19,2$ & $65,3 \pm 20,8$ & 0,05 \\
\hline Edad (años) Media \pm DE & $27,7 \pm 16,3$ & $26,7 \pm 15,6$ & $29,7 \pm 17,7$ & 0,20 \\
\hline Sexo masculino $n(\%)$ & $54(54)$ & $32(47,7)$ & $22(66,6)$ & 0,07 \\
\hline Temperatura axilar $\geq 380 \mathrm{C} n(\%)$ & $19(19)$ & $16(23,9)$ & $3(9,1)$ & 0,08 \\
\hline \multicolumn{5}{|l|}{ Durante el seguimiento } \\
\hline Definición clínica de denque hemorrágico n (\%) & $6(6)$ & $6(8,95)$ & 0 & 0,08 \\
\hline
\end{tabular}

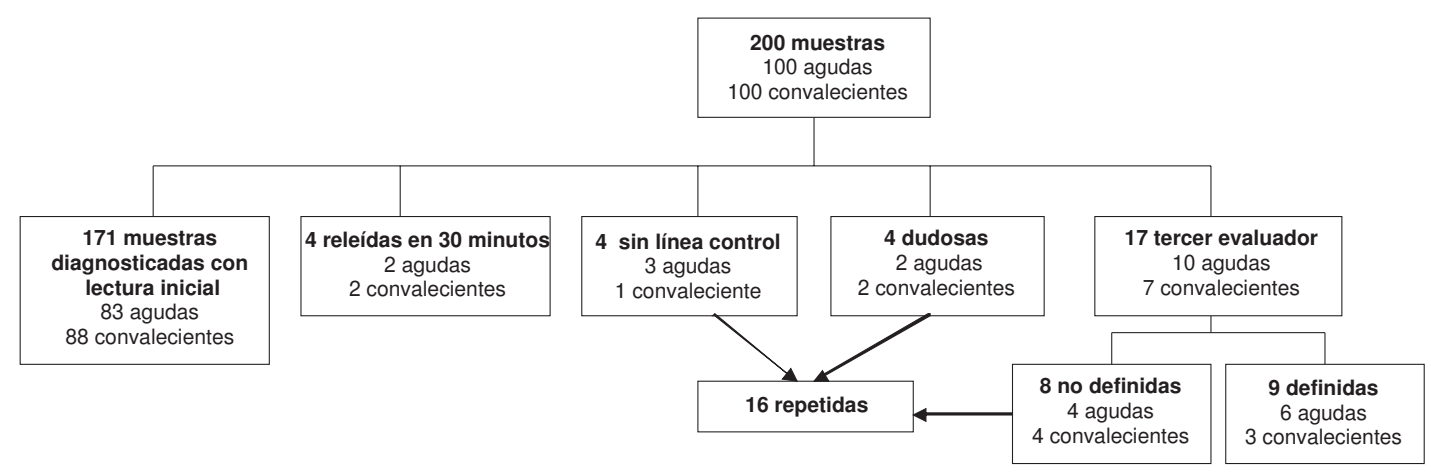

Figura 2. Descripción de la lectura de la prueba inmunocromatografía en casete para la inmunoglobulina M. 
de fase de convalecencia (después del séptimo día de inicio de la fiebre). Las variaciones de la especificidad en el tiempo fueron menores, aunque se observó una leve tendencia al descenso (cuadro 2).

De otra parte, la sensibilidad de la prueba de PANBIO en el suero de fase aguda fue más alta que la sensibilidad exhibida por la prueba ELISA, tanto en el grupo completo $(52,2 \%$ Vs. $32,8 \%)$, como en el grupo de menos de 72 horas de enfermedad $(29,4 \%$ Vs. $11,8 \%)$ y en el de más de 72 horas de enfermedad (60,0\% Vs. 40,0\%).

Además, la sensibilidad de la prueba de PANBIO® fue mayor cuando no se pudo aislar el virus en las muestras de fase aguda (58,3\% Vs. $36,8 \%)$; sin embargo, esto no fue estadísticamente significativo $(p=0,11)$ (cuadro 3). En el análisis de regresión logística se observó que el tiempo de evolución de la enfermedad al ingresar al estudio, fue mayor en los sueros agudos positivos para lgM en la prueba de inmunocromatografía, comparados con los que presentaron un resultado negativo. Igualmente, la detección de lgG por inmunocromatografía fue significativamente más frecuente en los sueros de fase aguda con prueba de casete positiva para IgM (cuadro 3).

Las razones de verosimilitud positivas, tanto para la muestra de fase aguda como para la de fase convaleciente, fueron mayores de 2 , pero las razones de verosimilitud negativas no eran muy bajas, excepto la de la convalecencia de 0,32 (cuadro 2).

\section{Discusión}

Durante el periodo del estudio, se captó una población de pacientes con síndrome febril agudo con una alta frecuencia de infección por dengue (67/100, 67,0\%). Los individuos con esta arbovirosis tenían un mayor tiempo de evolución de los síntomas en el momento de la captación. Considerando que los pacientes fueron captados cuando consultaron a los servicios de urgencias, este hallazgo sugiere que, en la población de estudio, el dengue presenta una mayor duración de los síntomas y, en especial, de la fiebre, en comparación con otras causas de síndrome febril agudo en nuestro medio.

De otra parte, la reproducibilidad entre observadores de la prueba PANBIO® de casete fue satisfactoria, aun cuando el nivel del entrenamiento de los observadores era diferente, lo que puede indicar que es una prueba de simple lectura y la haría fácilmente aplicable en distintos niveles de complejidad de atención en salud, incluidos aquéllos que no cuentan con personal de laboratorio clínico.

Los valores de especificidad encontrados en el presente estudio para la prueba PANBIO® de casete, estuvieron dentro de los valores

Cuadro 2. Características operativas de la prueba de lgM anti-dengue por inmunocromatografía (PANBIO®).

\begin{tabular}{|c|c|c|c|c|}
\hline \multirow[t]{2}{*}{ Característica } & \multicolumn{4}{|c|}{ Momento en el que se tomó la muestra de suero } \\
\hline & Aguda* & $\begin{array}{c}<72 \\
(n=35)\end{array}$ & $\begin{array}{c}\geq 72 \\
(n=65)\end{array}$ & $\begin{array}{c}\text { Convalecencia } \\
\text { ( } \geq 7 \text { días })\end{array}$ \\
\hline $\begin{array}{l}\text { Sensibilidad (\%) } \\
\text { (IC 95\%) }\end{array}$ & $\begin{array}{c}52,2 \\
(40,3-64,2)\end{array}$ & $\begin{array}{c}29,4 \\
(7,8-51,1)\end{array}$ & $\begin{array}{c}60,0 \\
(46,4-73,6)\end{array}$ & $\begin{array}{c}76,1 \\
(65,9-86,3)\end{array}$ \\
\hline Especificidad (\%) & 84,8 & 88,9 & 80,0 & 75,8 \\
\hline IC 95\% & $(72,6-97,1)$ & $(74,4-100)$ & $(59,8-100)$ & $(61,1-90,4)$ \\
\hline Valor diagnóstico positivo (\%) & 87,5 & 71,4 & 90,9 & 86,4 \\
\hline IC 95\% & $(77,3-97,7)$ & $(38,0-75,5)$ & $(81,1-100)$ & $(77,7-95,2)$ \\
\hline Valor diagnóstico negativo (\%) & 46,7 & 57,1 & 37,5 & 61,0 \\
\hline IC $95 \%$ & $(34,0-59,3)$ & $(38,8-75,5)$ & $(20,7-54,3)$ & $(46,0-75,9)$ \\
\hline Razón de verosimilitud positiva & 3,45 & 2,65 & 3,00 & 3,14 \\
\hline Razón de verosimilitud negativa & 0,56 & 0,79 & 0,50 & 0,32 \\
\hline
\end{tabular}

${ }^{*}$ Muestras tomadas antes de las 96 horas de haber iniciado la fiebre 
Cuadro 3. Factores determinantes del resultado de la prueba de IgM por inmunocromatografía en suero agudo de pacientes con dengue.*

\begin{tabular}{|c|c|c|c|c|c|c|}
\hline \multirow[t]{2}{*}{ Variable } & \multicolumn{2}{|c|}{$\begin{array}{l}\text { Análisis bivariado } \\
\text { IgM de fase aguda }\end{array}$} & \multirow[b]{2}{*}{$\begin{array}{c}\text { Valor } \\
\text { p } †\end{array}$} & \multicolumn{3}{|c|}{$\begin{array}{l}\text { Modelo de } \\
\text { regresión logística } \\
(n=67)\end{array}$} \\
\hline & $\begin{array}{c}\text { Positiva } \\
(n=35)\end{array}$ & $\begin{array}{c}\text { Negativa } \\
(n=32)\end{array}$ & & OR & (IC 95\%) & $\begin{array}{l}\text { Valor } \\
\mathbf{P}\end{array}$ \\
\hline Horas de enfermedad $\ddagger$ & $79,9 \pm 18,2$ & $66,7 \pm 18,1$ & $<0,01$ & 1,04 & $(1,01-1,08)$ & 0,01 \\
\hline Temperatura axilar $\geq 380 \mathrm{C}$ & $4(11,4)$ & $12(37,5)$ & 0,02 & 0,33 & $(0,08-1,38)$ & 0,13 \\
\hline IgG positiva en suero agudo & $15(42,9)$ & $4(12,5)$ & 0,01 & 5,75 & $(1,24-26,70)$ & 0,03 \\
\hline Aislamiento viral & $7(20,0)$ & $12(37,5)$ & 0,11 & 0,96 & $(0,25-3,73)$ & 0,96 \\
\hline
\end{tabular}

* Sólo se incluyen pacientes con dengue confirmado, comparando sus características de acuerdo con el resultado en la prueba de $\mathrm{PANBIO} \circledast$ realizada en suero de fase aguda de la enfermedad ( $\leq 96$ horas).

† Prueba T de Student para variables continuas y prueba exacta de Fisher para las dicotómicas.

¥ Se muestra la media \ la desviación estándar. Para las demás variables, se registra el número de pacientes y el porcentaje entre paréntesis.

reportados por la mayoría de los otros estudios que evalúan la prueba de $\mathrm{PANBIO} \AA^{\circledR}$ convencional (no casete). De acuerdo con un reciente metanálisis, la especificidad de estas últimas es, en promedio, de $88,0 \%$ y en la mayoría de los trabajos citados este valor oscila entre $78,0 \%$ y $94,0 \%(22)$.

La relación entre la sensibilidad de esta prueba y el tiempo de enfermedad, observada en el presente estudio, coincide con los resultados de otros trabajos en los que se han evaluado diferentes pruebas serológicas (22,24-26). En general, se ha descrito que la sensibilidad de la detección de IgM por cualquier método es muy baja en los primeros días de enfermedad $(25,26)$.

En el presente estudio, la sensibilidad en fase aguda de la prueba de casete, y en particular la obtenida en suero del cuarto día de enfermedad (52,2\% y 60,0\%, respectivamente), son superiores a las registradas en otros estudios con diferente metodología para la medición de IgM $(24,25)$, donde la sensibilidad en este mismo momento de la enfermedad es menor de $30 \%$ (24). Lo anterior sugiere que la prueba de inmunocromatografía de casete podría ser útil para el diagnóstico y la identificación temprana de los pacientes con dengue en áreas endémicas como la nuestra, donde la prueba evaluada también obtuvo un alto valor diagnóstico positivo (cuadro 2).
Además del tiempo de enfermedad, otros hallazgos asociados a un resultado positivo en la IgM en el presente estudio fueron la detección de la IgG y la ausencia de fiebre en el momento de la toma de la muestra. Estos resultados podrían explicarse por la aparición simultánea de ambas clases de anticuerpos, respuesta inmunológica que suele ocurrir con la mejoría clínica en las infecciones secundarias del dengue, propias de las zonas endémicas $(25,26)$.

Otro evento que ocurre con la disminución de la temperatura es la caída de la carga viral (27), por lo que cabe esperar una asociación negativa entre la detección del virus y la aparición de anticuerpos anti-dengue. Este supuesto podría explicar la posible asociación, no estadísticamente significativa, encontrada en el presente estudio, entre la detección de la IgM por inmunocromatografía y una menor frecuencia de aislamientos virales en el grupo de pacientes con dengue.

Además, con respecto a la aplicabilidad clínica, el valor de las razones de verosimilitud positivas de esta prueba, tanto en etapa aguda como de convalecencia, indican, de acuerdo con el nomograma propuesto por Fagan, que un sujeto febril con una prueba positiva elevaría la probabilidad previa a la prueba de tener dengue en un área endémica, de $40 \%$ (prevalencia) a $70 \%$ (28). De igual forma, la probabilidad previa a la prueba de $40 \%$ disminuiría a $20 \%$ cuando la 
prueba de IgM rápida de casete sea negativa en una muestra de fase convaleciente.

No obstante, cuando en una muestra aguda la prueba de lgM en casete es negativa, cabe considerar la posibilidad de un falso negativo, especialmente si ésta se realizó en una muestra tomada en las primeras 72 horas, cuando la sensibilidad es baja. En estos casos, realizar aislamiento viral o prueba de RT-PCR podrá esclarecer el diagnóstico.

Una limitación del presente estudio fue la imposibilidad de evaluar la validez de la lgG en la prueba de casete, debido a que no se cuenta con resultados de ELISA IgG en este grupo de pacientes. También debe considerarse que algunas posibles asociaciones, como la del aislamiento viral y el resultado de la prueba de IgM, pueden requerir una evaluación con un mayor número de pacientes.

En conclusión, la prueba casete de inmunocromatografía de $\mathrm{PANBIO} \cap$ para IgM mostró una alta reproducibilidad, buena especificidad, una sensibilidad aceptable entre las 72 y 96 horas de enfermedad y razón de verosimilitud positiva que eleva la probabilidad posterior a la prueba, razones por las cuales podría considerarse como una herramienta útil para la identificación temprana de pacientes con dengue que permita orientar el manejo del síndrome febril agudo en áreas endémicas.

\section{Conflicto de intereses}

Los autores declaramos no tener conflictos de intereses.

\section{Financiación}

Este estudio fue cofinanciado por Colciencias (proyecto: 1102-04-16433) y la Universidad Industrial de Santander.

\section{Referencias}

1. Gibbson RV, Vaughn DW. Dengue: an escalating problem. BMJ. 2002;324:33-42.

2. Pan American Health Organization, World Health Organization. 2008: Number of reported cases of dengue and dengue hemorrhagic fever (DHF), region of the Americas (by country and subregion). Figures for
2008 (to week noted by each country). Epidemiological Week/EW 53. Update: January 27, 2009. Fecha de consulta: febrero de 2009. Disponible en:http://www. paho.org/english/ad/dpc/cd/dengue-cases-2008.htm

3. Ministerio de la Protección Social, Instituto Nacional de Salud. Enfermedades de notificación obligatoria. Informe primer semestre de 2003. Sistema de Vigilancia en Salud Pública. Boletín Epidemiológico Semanal. Semana epidemiológica No. 26. Junio 22-28 de 2003. Fecha de consulta: febrero 2009. Disponible en:www. col.ops-oms.org/sivigila/2003/BOLE26_03.pdf

4. Gubler DJ. Dengue and dengue hemorrhagic fever. Clin Microbiol Rev. 1998;11:480-96.

5. Chairulfatah A, Setiabudi D, Ridad A, Colebunders R. Clinical manifestations of dengue haemorrhagic fever in children in Bandung, Indonesia. Ann Soc Belg Med Trop. 1995;75:291-5.

6. Harris E, Pérez L, Phares C, Pérez M, Idiaquez W, Rocha J, et al. Fluid intake and decreased risk for hospitalization dengue fever, Nicaragua. Emerg Infect Dis. 2003;9:1003-6.

7. Díaz-Quijano FA, Villar-Centeno LA, Martínez-Vega. Efecto de la administración de dipirona sobre la gravedad del dengue en una cohorte prospectiva. Enferm Infecc Microbiol Clin. 2005;23:593-7.

8. Martínez-Vega RA, Díaz-Quijano FA, Villar Centeno LA. Dificultad para el diagnóstico clínico temprano del dengue en un área endémica y su impacto sobre el manejo médico inicial. Rev Med Chile. 2006;134:1153-60.

9. Levett PN, Branch SL, Edwards CN. Detection of dengue infection in patients investigated for leptospirosis in Barbados. Am J Trop Med Hyg. 2000;62:112-4.

10. Watt G, Jongsakul K, Chouriyagune C, Paris R. Differentiating dengue virus infection from scrub typhus in Thai adults with fever. Am J Trop Med Hyg. 2003;68:536-8.

11. Seijo A, Cernigoi B, Deodato B. Dengue importado de Paraguay a Buenos Aires. Reporte clínico y epidemiológico de 38 casos. Medicina (Buenos Aires). 2001;61:137-41.

12. Pan American Health Organization. Dengue and dengue hemorrhagic fever in the Americas: guidelines for prevention and control. Scientific Publication No. 548. Washington, D.C.: PAHO; 1994. p. 3-22.

13. Dietz VJ, Gubler DJ, Rigau-Perez JG, Pinheiro F, Schatzmayr HG, Bailey R, et al. Epidemic dengue 1 in Brazil, 1986: evaluation of a clinically based dengue surveillance system. Am J Epidemiol. 1990;131:693-701.

14. Endy T, Chunsuttiwat S, Nisalak A, Libraty D, Green S, Rothman A, et al. Epidemiology of inapparent and symptomatic acute dengue virus infection: a prospective study of primary school children in Kamphaeng Phet, Thailand. Am J Epidemiol. 2002;156:40-50. 
15. Cuzzubbo AJ, Endy TP, Nisalak A, Kalayanarooj S, Vaughn DW, Ogata SA, et al. Use of recombinant envelope proteins for serological diagnosis of dengue virus infection in an immunochromatographic assay. Clin Diagn Lab Immunol. 2001;8:1150-5.

16. Groen J, Koraka P, Velzing J, Copra C, Osterhaus AD. Evaluation of six immunoassays for detection of dengue virus-specific immunoglobulin $\mathrm{M}$ and $\mathrm{G}$ antibodies. Clin Diagn Lab Immunol. 2000;7:867-71.

17. Wu SJ, Paxton H, Hanson B, Kung CG, Chen TB, Rossi C, et al. Comparison of two rapid diagnostic assays for detection of immunoglobulin $\mathrm{M}$ antibodies to dengue virus. Clin Diagn Lab Immunol. 2000;7:106-10.

18. Porter KR, Widjaja S, Lohita HD, Hadiwijaya SH, Maroef CN, Suharyono W, et al. Evaluation of a commercially available immunoglobulin $M$ capture enzyme-linked immunosorbent assay kit for diagnosing acute dengue infections.Clin Diagn Lab Immunol. 1999;6:741-4

19. Branch SL, Levett PN. Evaluation of four methods for detection of immunoglobulin $\mathrm{M}$ antibodies to dengue virus. Clin Diagn Lab Immunol. 1999;6:555-7.

20. Palmer CJ, King SD, Cuadrado RR, Perez E, Baum M, Ager AL. Evaluation of the MRL diagnostics dengue fever virus IgM capture ELISA and the PanBio Rapid immunochromatographic test for diagnosis of dengue fever in Jamaica. J Clin Microbiol. 1999;37:1600-1.

21. Lam SK, Devine PL. Evaluation of capture ELISA and rapid immunochromatographic test for the determination of $\operatorname{lgM}$ and $\operatorname{lgG}$ antibodies produced during dengue infection. Clin Diagn Virol. 1998;10:75-81.
22. Blacksell SD, Doust JA, Newton PN, Peacock SJ, Day NP, Dondorp AM. A systematic review and meta-analysis of the diagnostic accuracy of rapid immunochromatographic assays for the detection of dengue virus $\lg \mathrm{M}$ antibodies during acute infection. Trans R Soc Trop Med Hyg. 2006;100:775-84.

23. Innis BL, Nisalak A, Nimmannitya S, Kusalerdchariya S, Chongswasdi V, Suntayakorn S, et al. An enzymelinked immunosorbent assay to characterize dengue infections where dengue and Japanese encephalitis cocirculate. Am J Trop Med Hyg. 1989;40:418-27.

24. Díaz-Quijano FA, Martínez RA, Ocazionez RE, VillarCenteno LA. Evaluación de la prueba de lgM en suero agudo para el diagnóstico del dengue en un área endémica. Enferm Infecc Microbiol Clin. 2006;24:90-2.

25.Schilling S, Ludolfs D, van An L, Schmitz H. Laboratory diagnosis of primary and secondary dengue infection. J Clin Virol. 2004;31:179-84.

26. Vaughn DW, Green S, Kalayanarooj S, Innis BL, Nimmannitya S, Suntayakorn S, et al. Dengue in the early febrile phase: viremia and antibody responses.J Infect Dis. 1997;176:322-30.

27. Vaughn DW, Green S, Kalayanarooj S, Innis BL, Nimmannitya S, Suntayakorn S, et al. Dengue viremia titer, antibody response pattern, and virus serotype correlate with disease severity. J Infect Dis. 2000;181:2-9.

28. Fagan TJ. Letter. Nomogram for Bayes theorem. N Engl J Med. 1975;293:257. 